\title{
Fitometabolitos secundarios que inciden en el valor nutricional de Lotus corniculatus como forraje para rumiantes
}

\section{Secondary Fitometabolitos that affect the nutritional value of Lotus corniculatus as forage for ruminant animals}

\author{
Lado Fitometabolitos afetar o valor nutriciona \\ de Lotus corniculatus como forragem para ruminantes
}

\author{
Luz Elena Santacoloma Varón ${ }^{1}$ \& Jairo Enrique Granados Moreno ${ }^{2}$ \\ 1Zootecnista, Magister en Gestión ambiental para el Desarrollo Sostenible, Especialista en Nutrición Animal \\ Sostenible. ²Licenciado en Química, MSc en Ciencias Químicas, Magister en Docencia de la Química \\ 1,2Universidad Nacional Abierta y a Distancia (UNAD); Escuela de Ciencias Agrícolas, \\ Pecuarias y del Medio Ambiente (ECAPMA). Bogotá, Colombia. \\ 1'uz.santacoloma@unad.edu.co, 2jairo.granados@unad.edu.co
}

\section{Resumen}

En la sabana de Bogotá a 2600 msnm, temperatura $14^{\circ} \mathrm{C}$ y $1013 \mathrm{~mm}$ de precipitación se acondicionaron tres parcelas modificando las condiciones fisicoquímicas del suelo y se sembró la especie Lotus corniculatus para evaluar contenido de taninos condensados, taninos hidrolizables, fenoles totales, taninos que precipitan proteína, saponinas y alcaloides. El propósito fue determinar el efecto de las condiciones del suelo sobre la concentración de fitobiomoléculas secundarias. Se aplicaron técnicas analíticas e instrumentales de la AOAC para determinar la presencia de dichos fitometabolitos secundarios y los resultados obtenidos se sometieron a un análisis de varianza simple. Adicionalmente, se realizó un análisis de correlación múltiple, utilizando el coeficiente de Pearson. Se encontró que la producción de taninos condensados, taninos hidrolizables y taninos que precipitan proteína en el L. corniculatus, varía, con diferencias estadísticas altamente significati- vas $(P<0,01)$, de acuerdo al tipo de suelo. El contenido de fenoles totales, en el forraje no presentó diferencias significativas entre los tratamientos $(P>0,05)$. El nitrógeno amoniacal del suelo correlacionó de forma negativa y muy significativa con la concentración de taninos hidrolizables y con los taninos que precipitan proteína. Se identificó correlación negativa entre la capacidad de intercambio catiónica efectiva de los suelos y producción de taninos condensados y fenoles totales en la planta estudiada. Se identificó correlación entre el cambio de $\mathrm{pH}$ en el suelo y el contenido de taninos condensados y y altamente significativa con los taninos que precipitan proteína. Se concluye que la variación de las condiciones fisicoquímicas del suelo afectan la producción de metabolitos secundarios en $L$. corniculatus.

Palabras clave: capacidad de intercambio catiónico, conductividad eléctrica, nitrógeno amoniacal 


\section{Abstract}

In the highlands of Bogota at 2600 masl, temperature $14^{\circ} \mathrm{C}$ and $1013 \mathrm{~mm}$ of precipitation, three plots have been furbished by modifying the physico-chemical conditions of the soil and planted the species Lotus corniculatus to evaluate content of condensed tannins, hydrolysable tannins, phenols, tannins that precipitate protein, saponins and alkaloids. The purpose was to determine the effect of soil conditions on the concentration of secondary phytobiomolecules. Analytical and instrumental techniques of AOAC were applied to determine the presence of secondary phytometabolites and the obtained results were put through a simple variance analysis. Additionally a multiple correlation analysis was done, using the Pearson's coefficient. It was found that the production of condensed tannins, tannins and hydrolysable tannins that precipitate protein in L. corniculatus, varies, with highly different significant statistics according to the soil type. The content of total phenols, in the wrapping did not show significative differences among treatments $(P>0.05)$. The ammoniacal nitrogen of the soil correlated negatively and significantly with the concentration of hydrolysable tannins and with the tannins that precipitate protein. It was found negative correlation between the effective cationic exchange capacity of soils and production of condensed tannins and total phenols in the studied plant. A correlation between the change in $\mathrm{pH}$ in the soil and the content of condensed tannins and and hihgly significant with the tannins that precipitate protein was identified. It is concluded that the variation of the physico-chemical conditions of the soil affect the production of secondary metabolites in L. corniculatus.

Key-words: caption exchange capacity, electrical conductivity, ammoniacal nitrogen

\section{Resumo}

Na savana de Bogotá em 2600 metros acima do nível do mar, temperatura $14^{\circ} \mathrm{C}$ e 1013 milímetros de precipitação foram condicionadas três parcelas, modificando as condições físico-químicas do solo e foi plantada as espécie Lotus cornicul para avaliar o conteúdo de taninos condensadostaninos hidrolisáveis, fenóis totais, taninos que precipitam proteínas, saponinas e alcaloides. O objetivo foi determinar o efeito das condições do solo sobre a concentração de fitobiomoléculas secundárias. Aplicaram-se técnicas de análise instrumental AOAC para determinar a presença de tais fitometabolitos laterais e os resultados foram submetidos a uma análise de variância simples. Além disso, foi realizada uma análise de regressão múltipla por meio do coeficiente de Pearson. Verificou-se que a produção de taninos condensados, taninos hidrolisáveis e taninos que precipitam proteína em $L$. corniculatus varia, com diferenças altamente significativas $(P<0,01)$ de acordo com o tipo de solo. O teor de fenóis totais na forragem não foi significativamente diferente entre os tratamentos $(P>$ 0,05). O nitrogênio amoniacal no solo foi correlacionada negativamente e muito significativa com a concentração de taninos hidrolisáveis e taninos que precipitam proteína. Identificou-se correlação negativa entre a capacidade de troca catiônica efetiva dos solos e produção de taninos condensados e fenóis totais na planta estudada. Foi identificada correlação entre a alteração no pH do solo e o teor de taninos condensados e e alamente significativa com taninos que precipitam proteína. Concluiu-se que a variação das condições físico-químicas do solo afeta a produção de metabolitos secundários em L. corniculatus.

Palavras-chave: capacidade de troca catiônica, condutividade elétrica, nitrogênio amoniacal

\section{Introducción}

Las plantas sintetizan unos compuestos químicos denominados metabolitos secundarios que tienen varios propósitos como: la defensa contra hongos, bacterias y virus, protección contra la radiación ultravioleta y para evitar la deshidratación de sus tejidos; además por su sabor desagradable 
las plantas que poseen estos agentes presentan baja aceptabilidad para consumo por parte de los animales herbívoros, lo cual hace que disminuya su presión de pastoreo. Para Ramos, Frutos, Giraldez \& Mantecón (1998), ello se debe a que los metabolitos actúan con las micro-proteínas de la saliva o directamente con los receptores gustativos, y provocan la sensación de astringencia y consecuentemente la baja aceptabilidad de las plantas que los contienen.

Barahona, Theodoru, Morris, Owen \& Lascano (1997), plantea que la astringencia se define como la capacidad de los metabolitos de unirse a la proteína mediante enlaces de hidrógeno e interacciones hidrofóbicas principalmente. Debido a la habilidad de los taninos para precipitar proteínas salivales y para adherir a las membranas mucosas de la boca, un sabor astringente resulta del consumo de alimentos conteniendo los mismos, lo cual puede perjudicar la palatabilidad y por ende la aceptación de la ración

Siguiendo un criterio biosintético, los productos naturales secundarios podrían dividirse en tres grandes grupos de compuestos: nitrogenados, terpénicos y fenólicos. Los primeros, entre los que se encuentran los alcaloides, derivan principalmente de los aminoácidos. A partir de acetil-Co $A$, a través de la ruta del mevalonato se sintetizan los terpenos, mientras que según Troiani, Rosi, Angelini \& Ursini (2009), los metabolitos fenólicos provienen de dos vías diferentes: la del ácido shikímico y la de los policétidos o vía del malonato. Existe una gran variación cualitativa y cuantitativa en el contenido de metabolitos secundarios entre diferentes especies vegetales. La citada variabilidad se corresponde con los niveles de organización. Los distintos compuestos que puede producir una especie presentan una determinada distribución dentro de los órganos, tejidos y células de una planta, y ello responde frecuentemente a las influencias ambientales

Entre los metabolitos secundarios se encuentran los alcaloides, los cuales son bases nitrogenadas orgánicas, presentes en diversas familias de plantas, hongos, algas y otros vegetales inferiores. Uno o más átomos de nitrógeno está presente en amidas primarias, secundarias o terciarias y estas confieren usualmente basicidad a los alcaloides. Los aminoácidos precursores participan de la biosíntesis de los alcaloides, los principales son ornitina, lisina, ácido nicotínico, tirosina, triptófano, ácido antranílico e histidina. Algunas de las familias de plantas que tienen alcaloides son Papaveráceas (adormidera, amapola), Papilionáceas (retama), Ranunculáceas (acónitos), Solanáceas (tabaco, patata, belladona), Rubiáceas (quina, café) que no corresponden a los árboles forrajeros.

Por su parte Ashok, Vincent \& Nessler (200I), expresan que las saponinas son compuestos que en forma glucosídica (como esteroides reguladores del crecimiento) pueden generar en las plantas alto crecimiento y desarrollo, rápida recuperación ante la poda, y brotes abundantes. Lo anterior lo expresa también, Kumar (1997), quien expone que en dependencia de las concentraciones y las estructuras químicas específicas los compuestos saponínicos pueden constituir factores antinutricionales en rumiantes y monogástricos por conferirle a los forrajes un sabor amargo, provocar espumas consistentes, e interferir en la absorción de los alimentos. No obstante, Makkar, Blummel \& Becker (1997), plantean que también puede tener un efecto positivo en el metabolismo digestivo, al acomplejar otros metabolitos secundarios con características tóxicas

Otro grupo de singular importancia entre los metabolitos secundarios lo constituyen los taninos hidrolizables, para Leinmúller, Steingass \& Menke (2005), son compuestos de bajo peso molecular (500-3000), más solubles en agua que los condensados y pueden ser desdoblados bajo condiciones enzimáticas o ácidas a monosacáridos. Por presentar estas características, sus productos de degradación pueden ser absorbidos en el intestino delgado y ser potencialmente tóxicos para los rumiantes (Min, Atwood, McNabb, Molan \& Barry, 2005). Al respecto, Waghorn \& McNabb (2003); 
Rubansa, Shem, Otsyina, Bakenhesa, Ichihone \& Fujihara (2005), expresan que se han adelantado estudios tanto en ambientes in vivo como in vitro, acerca de la degradación de los taninos hidrolizables y sus efectos tóxicos y pocos estudios se han desarrollado en torno al suministro de nutrientes que éstos compuestos aportan. Es importante aclarar que por su rápida degradación en grupos fenólicos, no tienen capacidad de unirse posteriormente a las proteínas.

Por su parte, Márquez \& Suárez (2008), reportan que diversos factores influyen en la cantidad, clase de taninos que una planta puede sintetizar y en su concentración, estos factores son; la genética y especie de la planta, las condiciones climáticas como la temperatura, la fertilidad del suelo, el estado de desarrollo, el cultivo, el corte y el estado de crecimiento de la planta determinan la concentración de taninos en las plantas. Por tanto, para determinar dietas particulares e identificar los efectos nutricionales de los taninos en los animales, es necesario cuantificar estos factores Por su parte, los taninos condensados presentan mayor peso molecular y mayor complejidad en su estructura química, están presentes en casi todas las plantas particularmente en las dicotiledóneas, de las cuales hacen parte las leguminosas (Posada, Montoya \& Ceballos, 2005).

La distribución de este compuesto al interior de las plantas, varía entre especies y en la célula vegetal se encuentra en las vacuolas citoplasmáticas o en la pared celular. Según Norton (1999), los factores que inciden en el contenido de los taninos condensados en los árboles son: la genética de la planta, la especie, el grado de madurez, la estación climática, la humedad, el estado de crecimiento, la luminosidad, el corte y la defoliación por los herbívoros. Estos compuestos se caracterizan por altos pesos moleculares (entre 1900 y 28000) y no son fáciles de hidrolizar, por el contrario tienden a polimerizarse en productos insolubles especialmente en presencia de ácidos minerales para lo cual se hace necesario conocer la estructura química y la fisiología del animal objeto de estudio (Stürm, Tiemann, Lascano, Creuzer \& Hess, 2007; y Bueno, Vitti, Louvandini \& Abdala 2008). reportan que el tipo y el contenido de taninos, están influenciados por el genotipo de la planta (la especie y la variedad), las características ambientales (radiación solar y disponibilidad de agua), la velocidad de crecimiento, la madurez, la condición nutricional del suelo, la depredación y las enfermedades.

En el mismo sentido, Otero \& Hidalgo (2004), reportan que la concentración de taninos en los forrajes, presenta gran variación según las condiciones del ambiente en cuanto a temperatura, humedad y fertilidad de los suelos en los que se desarrollan, exponen que la concentración es mayor en las especies que prosperan en suelos agrícolas pobres o de baja calidad, tal es el caso de las regiones tropicales y subtropicales. Un ejemplo de esto lo constituye la especie Acacia segal (Sesbania goetsia) con un rango de concentración amplio (4,8-8 \% de MS). Un factor ambiental de alta incidencia en la concentración de taninos es la fertilidad del suelo, al respecto, Barry \& Mc Nabb 1989, citado por Berard, et al., 2011 reportaron una concentración de taninos condensados de 8 al 11\% en Lotus pedunculatus creciendo en suelos ácidos sin aplicaciones de fertilizantes, mientras que en condiciones de mejor calidad evidenciaron valores tan solo de 2 al $3 \%$. Otro factor ambiental importante es la baja disponibilidad de agua, la cual contribuye a la producción de taninos condensados; debido a que las plantas al encontrarse en periodo de escasez de agua, cierran sus estomas y restringen el proceso de fotosíntesis.

Adicionalmente, entre las plantas forrajeras de trópico alto, se ha encontrado que la del género Lotus, en especial el L. Corniculus contienen taninos condensados (TC) en sus hojas, y también se ha detectado que la presencia de estos compuestos evita el meteorismo en rumiantes. De la misma forma, se ha determinado que niveles bajos de taninos condensados en la dieta, optimizan la utilización de proteína sin afectar el consumo ni la digestibilidad de los carbohidratos. 


\section{Los metabolitos y su efecto en la utilización del forraje}

En animales rumiantes se ha evidenciado un descenso en la digestibilidad ruminal de la materia orgánica de forrajes con altos contenidos de metabolitos secundarios particularmente de taninos; para Leinmúller, et al. (1995), lo anterior se debe a que algunos de éstos exhiben un efecto inhibidor sobre actividad de las enzimas tales como proteasas, zimógenos, lipasa, alfa-amilasa, celulasa, betaglucosidasa y ureasa. Según Norton (1999), los taninos también inhiben la degradación de la proteína dietaria en el rumen disminuyendo las concentraciones de amonio, lo cual sugiere una inhibición de las enzimas proteolíticas a este nivel.

Los taninos condensados son bastante activos en la nutrición de rumiantes, debido a su alta capacidad de unirse a proteínas del forraje en el rumen después de su proceso de masticación (Andrabi, Ritchie, Stimson, Horadagoda, Hyde \& McNeil, 2005; Stürrn et ál., 2007) y a su capacidad para disminuir la degradación de proteína en el rumen, cuando sus concentraciones son óptimas (Provenza, Burrit, Perevolotsky \& Silanokove, 2000). Al respecto Rojas et al. (2006), expresan que cuando las concentraciones de proteína cruda en la dieta son bajas y las de fibra altas, los taninos condensados pueden generar efectos desfavorables.

Este aspecto resulta a la postre benéfico si se tiene en cuenta la propensión de los taninos condensados a formar complejos químicos con las proteínas, protegiéndolas de la degradabilidad ruminal y mejorando su utilización en el tracto digestivo posterior, así mismo, permitiría a los animales una mayor disponibilidad de aminoácidos como consecuencia del sobrepeso generado (Galindo, Rosales, Murgueitio \& Larrahondo, 1989). En este mismo sentido Posada, et al. (2005) expresan que los taninos condensados ligan las proteínas de las plantas reduciendo su solubilidad y degradación por parte de los microorganismos ruminales. Lo anterior, tiene como consecuencia, un mejoramiento del flujo de proteínas al intestino y un incremento en la disponibilidad y absorción de aminoácidos esenciales. Sustentando esta afirmación Barry \& Mc Nabb (1999) observaron que el incremento en la concentración de taninos condensados de Lotus curniculatus y de Lotus pedunculatus, redujo la tasa de degradación y solubilización de la fracción 1 de la proteína en el rumen y aumentó el flujo de nitrógeno no amoniacal hacia el duodeno.

Al respecto, Márquez \& Suárez (2008), exponen que cantidades moderadas de taninos condensados producen efectos benéficos sobre el metabolismo de las proteínas en rumiantes, porque reducen la degradación de la dieta proteínica en el rumen e incrementan la absorción de aminoácidos en el intestino delgado. Estos efectos de los taninos condensados sobre los procesos digestivos en rumiantes, dependen de su capacidad para adherir a diferentes moléculas y ello depende de su estructura química y de las moléculas presentes en los procesos digestivos como proteínas, celulosa, polisacáridos, entre otros. Estos argumentos también son expuestos por Waghorn, Redd \& Ndlovu (1997), en Otero \& Hidalgo (2004), en el sentido de que las altas concentraciones (5-10\% de la materia seca), deprimen el consumo y la digestibilidad del forraje, en tanto que menores concentraciones $(2-4 \%$ de la materia seca), podrían disminuir las pérdidas de la proteína de la ingesta producida por la proteólisis por los microorganismos del rumen e incrementar la absorción intestinal de las proteínas.

Nastis \& Malechek (1988), citado por Posada et al (2005), al experimentar con roble (Quercus spp), con una concentración de taninos de $8.7 \%$, observaron que la actividad in-vitro de la pepsina se redujo, lo cual ocurrió con mayor intensidad en la medida en que esta especie estuvo presente en mayor porcentaje que la alfalfa (Medicago sativa), la cual tiene un $1 \%$ de taninos. Los mismos autores llegaron a la conclusión que los taninos de la hoja de roble madura fueron más efectivos en reducir la actividad de la enzima que los presentes en la hoja compuestos en la saliva, lo cual afecta la digestión de los rumiantes con consecuencias negativas sobre el crecimiento de los animales. 
Otero \& Hidalgo (2004), reportan que algunas especies forrajeras como Lotus pedunculatus variedad Maku y Lotus corniculatus variedad Goldie han provocado mejoras en aspectos productivos (aumentos en la ganancia de peso) y sanitarios cuando intervienen los taninos, debido probablemente a una mayor disponibilidad proteica inmadura. Del mismo modo Caviedes, Pabón \& Carulla (2011), reportan que la presencia de metabolitos secundarios modifican los patrones de biohidrogenación ruminal al incrementar la producción de precursores de Acido Linoleico Conjugado (ALC). En este sentido Min et al. (2005), encontraron que los taninos tienen un efecto sobre la disminución de cepas de Butyrivibrio fibrisolvens, una de las especies que más incide en la biohidrogenación y por tanto la oferta de forrajes con estos metabolitos modifica los patrones de fermentación de los lípidos mejorando el contenido de ALC. Así mismo, Khiaosa-Ard et al. (2009), reportaron que la incorporación de leguminosas taniferas como el Lotus corniculatus, tiene un efecto benéfico sobre el contenido de ácidos grasos de la leche. Sin embargo, Márquez \& Suarez (2008), refieren que los efectos negativos de los taninos condensados se relacionan con la disminución del consumo de alimentos, debido probablemente a la reducción de la palatabilidad ocasionada por los efectos astringentes de estos.

Por tanto, el presente trabajo se propone determinar el efecto de la fertilidad del suelo en el contenido de metabolitos secundarios de la leguminosa Lotus corniculatus, que pueda afectar el valor nutricional de esta especie en su utilización como forraje para animales rumiantes. Para estos propósitos se establecen análisis de correlación entre los principales indicadores de fertilidad del suelo y el contenido de fenoles totales, taninos hidrolizables, taninos condensados, taninos que precipitan proteína, saponinas y alcaloides.

\section{Metodología}

Localización: La experiencia ha tenido en cuenta dos lugares de trabajo:

- En campo: parcelas ubicadas en la sabana de Bogotá

- En laboratorio: laboratorio particular de suelos y laboratorio de Nutrición, sede Nacional José Celestino Mutis de la UNAD.

\section{Trabajo en campo:}

El trabajo fue realizado en la ciudad de Bogotá ubicada a 2600 metros sobre el nivel del mar y una temperatura promedio de 14 grados centígrados. Para ello, se utilizaron tres parcelas de $2 \mathrm{~m} \times 2 \mathrm{~m}$, (Figura 1) de las cuales se tomaron muestras de suelo, utilizando el método de zig-zag para cuantificar en ellas variables de fertilidad como: $\mathrm{pH}$, carbono orgánico, materia orgánica, nitrógeno total, nitrógeno disponible, capacidad de intercambio catiónico y conductividad eléctrica. Las muestras de suelo fueron empacadas en bolsas negras, rotuladas y transportadas hasta un laboratorio particular, ubicado en la ciudad de Bogotá D.C, donde se realizaron los respectivos análisis fisicoquímicos.

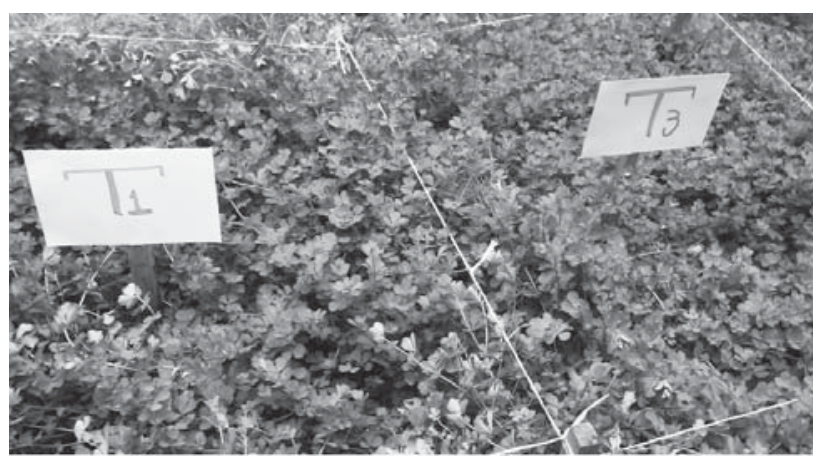

Figura 1. Aspecto de las parcelas de Lotus corniculatus 


\section{Toma de muestras}

Las muestras del forraje en cada parcela, se tomaron específicamente de hojas, peciolo y tallos de Lotus curniculatus, a los 6 meses de edad, cortados a $4 \mathrm{~cm}$ del suelo, en forma manual, a partir de 10 plantas por parcela seleccionadas al azar, cuidándose de eliminar el efecto de borde (Figura 2). Las muestras fueron empacadas en bolsas negras, rotuladas adecuadamente, para ser transportadas hasta el laboratorio de nutrición de la sede nacional José Celestino Mutis de la UNAD, en la ciudad de Bogotá D.C, donde se realizaron los respectivos análisis fitoquímicos.

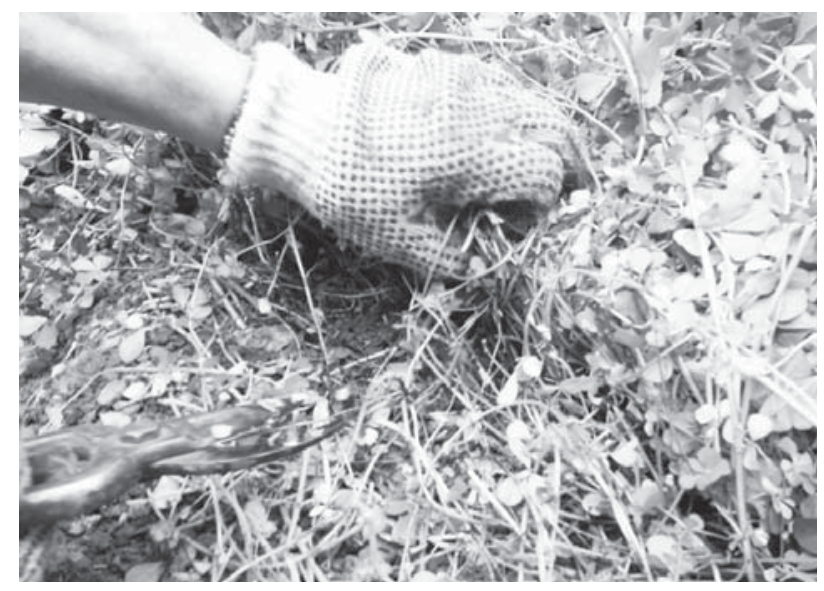

Figura 2. Recolección de la muestra de forrajes

\section{Análisis de laboratorio}

Una vez ingresadas las muestras al laboratorio fueron sometidas a proceso de secado al horno, a una temperatura de $105^{\mathrm{a}} \mathrm{C}$ durante 6 horas para el posterior tamizaje fitoqumico y se enfocó el análisis en los siguientes fitometabolitos (ver Tabla 1) taninos hidrolizables, taninos condensados, taninos que precipitan proteínas, fenoles totales y saponinas, los cuales han sido reportados en investigaciones preliminares.

\section{Metabolitos analizados}

\section{Taninos hidrolizables (TH)}

La extracción de taninos hidrolizables se realizó, utilizado como disolventes agua (temperatura ambiente), metanol en extracción soxleth, en diferentes proporciones Beecher et al., 2004; Mueller-Harvey, 2001). Es importante tener presente que los compuestos extraíbles poseen pesos moleculares bajos o medios (de monómeros a decámeros) mientras que los no extraíbles son compuestos con un peso molecular elevado (5000 unidades o mayores).

Los Polifenoles Hidrolizables (PH) son extraídos del residuo por tratamiento con metanol) a temperatura ambiente durante 4 horas con agitación (Hartzfeld, Forkner, Hunter \& Hagerman, 2002) obteniéndose un hidrolizado que después es usado para la cuantificación de compuestos polifenólicos. La reacción con las sustancias reductoras, produce coloración azul y permite su lectura en un espectrofotómetro, la cual se realizó con UV-VIS a longitud de onda de $700 \mathrm{~nm}$. La coloración fue cotejada con una solución de ácido tánico comercial (curva de calibración).

\section{Proantocianidinas o taninos condensados (TC)}

Los taninos condensados conocidos genéricamente como poliflavonoides o proantocianidinas están constituidos por flavonoides con diferentes grados de condensación (flavan-3-ol y flavan-3,4diol) así como otros flavonoides análogos, carbohidratos y trazas de amino-ácidos. La extracción se llevó a cabo con solventes orgánicos éter-acetona (Terril, Rowan, Douglas \& Barry, 1997) obteniéndose en el extracto una mezcla de diferentes compuestos polifenólicos. Los taninos condensados se obtuvieron por tratamiento del residuo con una solución de butanol $/ \mathrm{HCl}$, produciéndose el paso de proantocianidinas de alto peso molecular a unidades más sencillas en forma de cationes flavilium (solución coloreada de antocianidinas). 
Las reacciones químicas generan cambios en la coloración, y ésta fue realizada en equipo UV a 760 $\mathrm{nm}$, donde la muestra es comparada con una curva de calibración corrida previamente con cantidades conocidas de ácido tánico y por cálculos se obtiene el resultado en porcentaje. Se caracterizaron las fracciones de taninos condensados libres y los complejos formados con la proteína y la fibra, según Terrill et al. (1997). La concentración de polifenoles totales se determinó según Makkar \& Goodchild (1996), utilizando el reactivo de Folín-Ciocalteu.

\section{Fenoles totales (FT)}

La extracción se obtuvo mediante solución acuosa y posterior filtración con papel filtro, el extracto obtenido se mezcló con solución de carbonato de sodio al $20 \%$ y el reactivo específico de Folin-Ciocalteu. De igual manera, se realizó la curva de calibración con soluciones stock, de ácido tánico. Para la determinación se utilizó un método espectrofotométrico según Waterman \& Mole (1995), con lectura de absorbancia a $720 \mathrm{~nm}$, agregando como colorantes: cloruro férrico $0.1 \mathrm{M}$ y ferricianuro de potasio $0,008 \mathrm{M}$ al extracto metanólico de la muestra.

\section{Taninos que precipitan proteínas (TPP)}

Los taninos que precipitan proteína, forman complejos tanino-proteína estructurados por enlaces de hidrógeno entre los grupos fenólicos de taninos y los grupos cetoamida de las proteínas, así mismo, por interacciones hidrofóbicas entre los anillos aromáticos de los taninos y las regiones hidrofóbicas de las proteínas. El proceso de formación del complejo es normalmente reversible y tanto las proteínas como los taninos pueden, en principio, ser recuperados intactos. Los factores que determinan la relativa afinidad de las proteínas por los taninos son el tamaño de la molécula, su composición aminoacídica y el pH.
Para obtener estos compuestos (TPP), el extracto metanólico se trató con albúmina en buffer, se adicionó dodecil sulfato de sodio (SDS), ferrocianuro de potasio y cloruro acetatos, posteriormente férrico para desarrollar color. Más adelante se realizó la lectura espectrofotométrica para medir la absorbancia. Al respecto, es importante aclarar que estudios desarrollados in vitro, y corroborados en experimentos in situ, han demostrado que los TPP son el principal indicador que se debe considerar para estimar la acción detrimental de los compuestos fenólicos en la fisiología digestiva de los rumiantes; ya que en dependencia de la estructura de los FT, TT y TC estos pueden precipitar o no las proteínas (Makkar et al., 1997).

\section{Saponinas}

Se realizó un análisis cualitativo de estos componentes de la siguiente forma: a un mililitro del extracto metanólico acuoso, se agregaron 4 mililitros de agua destilada, luego se agitó vigorosamente por 1 minuto y se dejó reposar 5 minutos con el fin de detectar la presencia de espuma, cuando esta se presentó, entonces se midió su altura en milímetros con una regla. Aunque no todas las Saponinas y los alcaloides son metabolitos deletéreos, cuando los niveles son cuantiosos actúan como inhibidores del consumo; tienen propiedades espumantes, presentan sabor amargo y constituyen potentes tóxicos en el metabolismo digestivo (García et al., 2007).

Alcaloides: el extracto fue sometido a un tratamiento con amoníaco, luego se calentó hasta un punto cercano a la evaporación, se le adicionó ácido acético glacial y el reactivo dragendorff, de tal forma que al presentarse en la solución un color rojo o rosado, daba prueba positiva para alcaloides. 
Tabla 1. Resumen de técnicas utilizadas en la obtención de metabolitos secundarios

Metabolitos

Taninos condensados (TC)

Taninos hidrolizables

Fenoles Totales

Taninos que precipitan proteína

Saponinas

Alcaloides

\section{Técnica}

Espectrofotometría con $\mathrm{n}$-butanol $-\mathrm{HCl}$

Espectrofotometría UV-VIS a longitud de onda de $700 \mathrm{~nm}$

Folin-Ciocalteu

Albúmina, dodecil sulfato de sodio (SDS), ferrocianuro de potasio y cloruro férrico para desarrollar color

Extracto metanólico acuoso, agua destilada, detección de presencia de espuma

Amoníaco, adición de ácido acético glacial y reactivo Dragendorff

\section{Diseño experimental}

- Se aplicó un diseño completamente al azar, con tres tratamientos, tres réplicas por tratamiento y una muestra por repetición para un total de 9 unidades experimentales.

- El modelo matemático fue el mostrado en la euación 1

$$
\Psi=\mu+\alpha+\varepsilon,
$$

$\psi=$ Representa el contenido de los diferentes metabolitos evaluados en el laboratorio

$\mu=$ Media poblacional;

$\alpha=$ Representa los tratamientos $\left(\mathbf{T}_{1}\right.$ : parcela testigo en la cual no se realizó ningún proceso de fertilización y $\mathbf{T}_{2}$ representa la parcela 2 correspondiente al suelo con adición de arena y $\mathrm{T}_{3}$ a la parcela con fertilización biológica (lombricompost).

$\varepsilon=$ representa el efecto de las réplicas ó error experimental del análisis.

\section{Análisis estadístico}

Las posibles diferencias estadísticas entre tratamientos, se evaluaron, utilizando elementos de la estadística descriptiva e inferencial como:

- Media, desviación estándar, coeficiente de variación y límites de confianza de la media.

- Análisis de varianza(ANAVA) y prueba de comparación múltiple de medias por el método Duncan

- Matriz de correlación de Pearson, con las diferentes variables evaluadas.

- Estos análisis se apoyan con los Software estadísticos .SPSS y S.A.S.

\section{Resultados y discusión}

Análisis de suelo: los resultados obtenidos en el análisis de suelo en el cual se tuvieron en cuenta indicadores como $\mathrm{pH}$, textura, materia orgánica, carbono orgánico, capacidad de intercambio catiónico, Nitrógeno total y fósforo son reportados en la Tabla 2. 
Tabla 2. Análisis de suelo realizado a los tres tratamientos

\begin{tabular}{lccccccc}
\hline \multicolumn{1}{c}{ Tratamiento } & $\mathbf{p H}$ & Textura & $\begin{array}{c}\text { Materia } \\
\text { orgánica }\end{array}$ & $\begin{array}{c}\text { Carbono } \\
\text { orgánico }\end{array}$ & $\mathbf{C l C}$ & $\begin{array}{c}\text { Nitrógeno } \\
\text { Total }\end{array}$ & Fosforo \\
$\begin{array}{l}\text { Tratamiento 1 } \\
\text { Suelo original }\end{array}$ & 6.84 & Franca & 2.26 & 1.31 & 19 & 77 \\
$\begin{array}{l}\text { Tratamiento 2 } \\
\text { Suelo y arena }\end{array}$ & 7.18 & $\begin{array}{c}\text { Franca- } \\
\text { arenosa }\end{array}$ & 1.43 & 0.83 & 17 & 85 \\
$\begin{array}{l}\text { Tratamiento 3 } \\
\text { Suelo y compost }\end{array}$ & 6.95 & Franca & 6.19 & 3.59 & 21 & 133 \\
\hline
\end{tabular}

En la Tabla 2 se observa que en el tratamiento 3, al cual se le adicionó compost presenta un pH más cercano al neutro, este tratamiento también presenta unos contenidos más altos de materia orgánica, carbono orgánico, capacidad de intercambio catiónico y fósforo que los tratamientos 1 y 2 . Por su parte el suelo más arena, correspondiente al tratamiento 2 presenta un menor contenido de materia orgánica, carbono orgánico, y capacidad de intercambio catiónico.

\section{Taninos condensados en los forrajes}

En la determinación de taninos condensados en el forraje de Lotus corniculatus se encontró, que en el tratamiento 2, se presentó una mayor concentración de estos metabolitos y ello permite deducir que la disminución de nutrientes disponibles en el suelo, pudo haber generado estrés en la planta y como consecuencia de ello una mayor producción de taninos condensados. En el tratamiento 3, el cual presenta mayores niveles de fertilidad en el suelo, se observa menor contenido de metabolitos en el forraje estudiado. Este comportamiento coincide con lo planteado por Waghorn, \& McNabb (2003) en el sentido de que las concentraciones de taninos condensados en hojas de Lotus corniculatus, aumentaron cuando las condiciones del suelo fueron pobres. Por su parte Barry \& McNabb (1999) encontraron que la concentración de taninos condensados en Lotus pedunculatus fue de $11.8 \%$ de la materia seca, cuando se cultivó en suelos ácidos, sin aplicación de fertilizantes y de $2.3 \%$ de MS cuando se cultivó en suelos con alta fertilidad. Lo anterior se explica, porque la concentración de iones en la solución junto con las propiedades físico-químicas, pueden afectar la movilización de nutrientes desde el suelo a la planta (Jungk 2001). Este comportamiento se puede apreciar en la Figura 3.

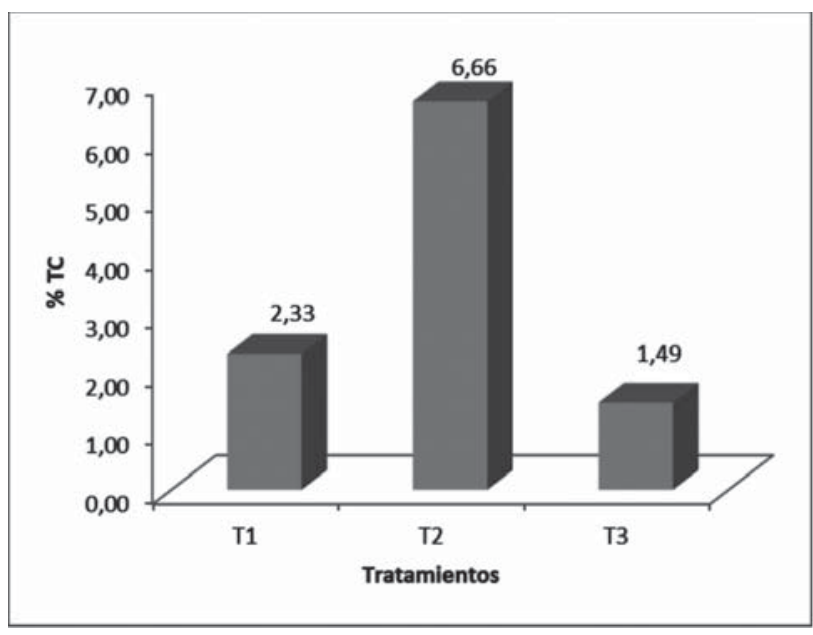

Figura 3. Concentración (\%) de taninos condensados en el forraje de Lotus corniculatus en los tres tratamientos

Otros factores ambientales también afectan el contenido de taninos condensados, al respecto, Robbins, Bavage, Allison, Davies, Hauck \& Morris (2005) encontraron que los niveles de taninos se incrementaron en hojas y tallos de Lotus corniculatus al duplicarse el $\mathrm{CO}_{2}$ ambiental

Cuando se realizó el análisis de varianza simple para los promedios de taninos condensados se encontraron diferencias estadísticas altamente significativas $(P<0,01)$, entre los tratamientos, lo cual se puede atribuir a las diferencias de las propiedades fisicoquímicas existentes en cada parcela. 


\section{Taninos hidrolizables en forrajes}

En los taninos hidrolizables se encontró un comportamiento similar al de los taninos condensados y fue posible establecer que en el tratamiento 2 se presentaron mayores contenidos de estos metabolitos, como se observa en la Figura 4:

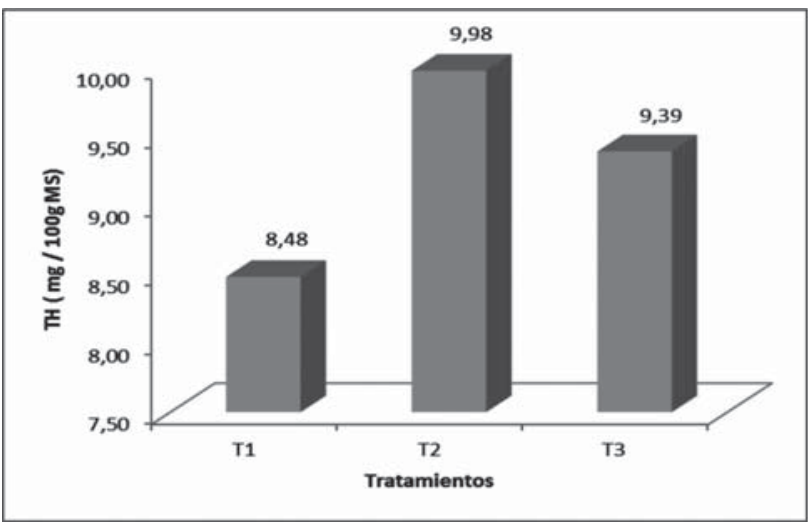

Figura 4. Concentración (\%) de taninos hidrolizables en el forraje de Lotus corniculatus en los tres tratamientos
El comportamiento presentado con los taninos hidrolizables, también es posible atribuirlo a las condiciones físicas del suelo, ya que con la adición de arena, predominan condiciones de baja retención de humedad, y ello afecta la alimentación radicular y produce trastornos severos en el desarrollo vegetal. Esta situación pudo haber generado condiciones para un incremento en los niveles de metabolitos secundarios como son los taninos hidrolizables. Lo anterior es coherente con lo expresado por Thevenot, Dignac \& Rumpel, (2010), cuando manifiesta que la concentración de metabolitos secundarios depende de diversos factores, como $\mathrm{pH}$ y textura. Al respecto, Vilela, Gonzalez-Paleo \& Ravetta (2011), señalan que las plantas en zonas áridas desarrollan enorme diversidad de metabolitos secundarios debido a las presiones de selección y a las relaciones de disponibilidad de recursos.

Tabla 3. Resumen de estadística descriptiva taninos hidrolizables

\begin{tabular}{lcccccc}
\hline Grupos & Cuenta & Suma & Promedio & Varianza & SD & $\%$ CV \\
T1 & 3 & 25,43902 & 8,4796748 & 0,341661709 & 0,58451836 & 6,89 \\
T2 & 3 & 29,9268293 & 9,97560976 & 0,000594884 & 0,02439024 & 0,24 \\
T3 & 3 & 28,1707317 & 9,3902439 & 0,0446163 & 0,21122571 & 2,25 \\
\hline
\end{tabular}

Como se puede apreciar en la Tabla 3 los bajos coeficientes de variabilidad muestran una buena confiabilidad en el valor reportado de promedios del análisis de taninos hidrolizables y al realizar el análisis de varianza simple para los promedios de $\mathrm{TH}$ se encontraron diferencias estadísticas altamente significativas $(P<0,01)$, entre los tratamientos, lo cual se puede atribuir a las diferencias de las propiedades fisicoquímicas existentes en cada parcela.

\section{Fenoles totales (FT)}

En los fenoles totales, se encontró un comportamiento similar al de los taninos condensados e hidrolizables, sin embargo cuando se realizó el análisis de varianza simple para los promedios de FT, no se encontraron diferencias estadísticas altamente significativas $(P>0,05)$, entre los tratamientos, lo cual indica que los tipos de suelo de cada parcela no afectaron la producción de estos metabolitos. Lo anterior, no coincide con lo planteado por García et al. (2007), quienes evaluaron el efecto de la fertilización orgánica en el perfil polifenólico de Morus alba, y encontraron interacciones significativas entre los factores fertilización y variedad para los fenoles totales y las cumarinas con un nivel de significancia de $(P<0,05)$, el trabajo citado encontró que los contenidos de fenoles 
totales y cumarinas tienden a disminuir con el incremento de niveles de fertilización.

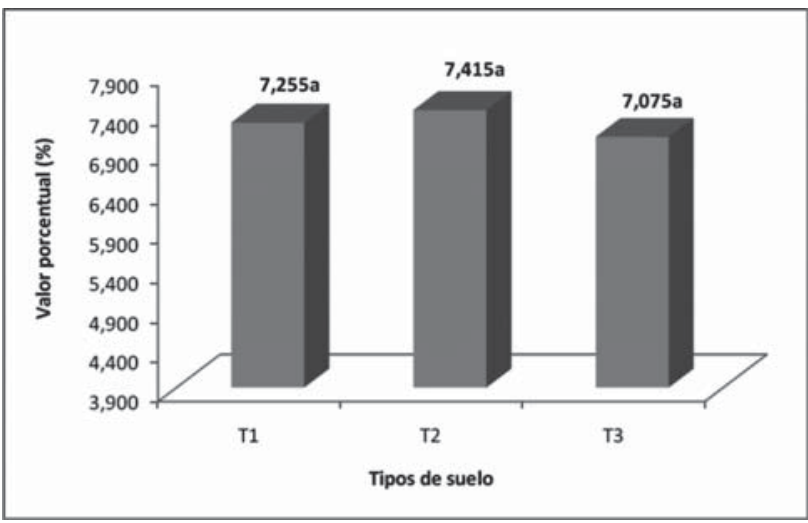

Figura 5. Concentración (\%) de fenoles totales en el forraje de Lotus corniculatus en los tres tratamientos

\section{Taninos que precipitan proteína (TPP)}

Dado que los taninos condensados tienen la capacidad para adherirse a otras moléculas, como proteínas, polisacáridos, minerales, carbohidratos, celulosa, células de las membranas bacterianas y enzimas involucradas en la digestión de los compuestos antes mencionados, la unión con proteínas es una de las características que presentan mayor impacto en la nutrición animal. Por lo anterior se determinó la concentración de TPP, y se encontró que en el tratamiento 2 se presenta mayor concentración de TPP, y ello coincide con unas características fisicoquímicas en el suelo, inferiores a las de los demás tratamientos.

Cuando se realizó el análisis de varianza simple para los promedios de TPP se encontraron diferencias estadísticas altamente significativas $(P<$ 0,01 ), entre los tratamientos, lo cual se puede atribuir a las diferencias de las propiedades fisicoquímicas existentes en cada parcela. Lo anterior es coherente con lo expresado por Posada, Montoya \& Ceballos (2005), en el sentido de que la fertilidad del suelo es un factor interviniente en el contenido de taninos en las especies vegetales.
En la Figura 6 se observa el comportamiento de los taninos que precipitan proteína en el forraje de Lotus corniculatus en los tres tratamientos

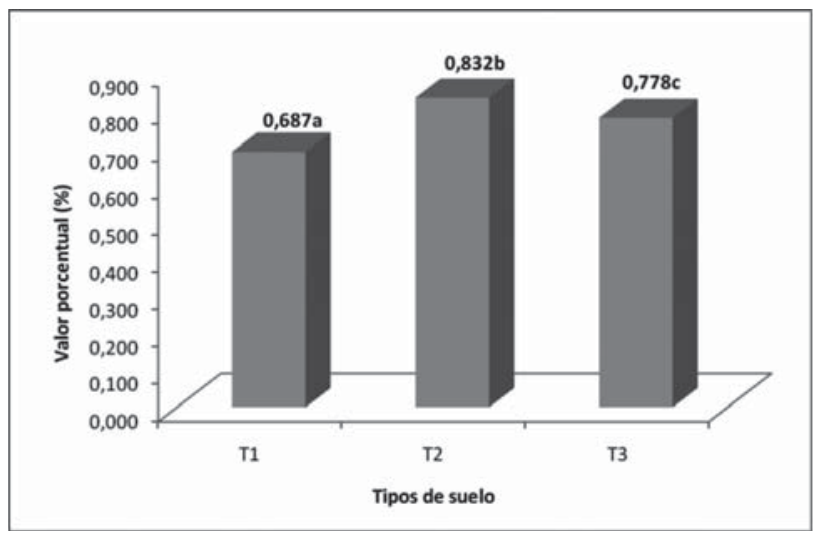

Figura 6. Concentración de Taninos que Precipitan Proteína (TPP) en los tres tratamientos

La presencia de TPP es particularmente importante en la utilización del Lotus corniculatus como forraje para alimentación de rumiantes, si se tiene en cuenta que Wang, Douglas, Waghorn, Barry, Foote \& Purchas (1996), al evaluar la mejor producción de ovejas que pastorearon este forraje en contraste con la alfalfa, encontraron mejores rendimientos en el primero, debido a que los TC del Lotus corniculatus complejaron las proteínas y facilitaron la absorción de aminoácidos específicos (valina, leucina, arginina y lisina) en el intestino delgado. Así mismo, hubo una menor concentración de ácidos grasos volátiles, producto de una menor desaminación a nivel ruminal.

\section{Análisis de correlación entre las varia- bles del suelo y los metabolitos secundarios}

Los resultados obtenidos en el estudio de la correlación entre las variables del suelo y los metabolitos secundarios se pueden observar en la Tabla 4: 
Tabla 4. Análisis de coeficientes de correlación-significancia estadística

\begin{tabular}{|c|c|c|c|c|c|c|c|c|c|}
\hline & $T C$ & $\mathrm{TH}$ & $F T$ & $T P P$ & $\mathrm{~N}-\mathrm{NH} 4$ & $N-N O 3$ & CIC E & $P H$ & $C E$ \\
\hline TC & 1,000 & & & & & & & & \\
\hline $\mathrm{TH}$ & NS & 1,000 & & & & & & & \\
\hline $\mathrm{FT}$ & ${ }^{* *}(+)$ & NS & 1,000 & & & & & & \\
\hline TPP & NS & ${ }^{* *}(+)$ & NS & 1,000 & & & & & \\
\hline $\mathrm{N}-\mathrm{NH} 4$ & NS & ${ }^{* *}(-)$ & NS & $* *(-)$ & 1,000 & & & & \\
\hline $\mathrm{N}-\mathrm{NO} 3$ & NS & NS & NS & NS & ${ }^{*}\left(\_\right)$ & 1,000 & & & \\
\hline $\mathrm{CICE}$ & ${ }^{* *}(-)$ & NS & $* \star(-)$ & NS & NS & NS & 1,000 & & \\
\hline $\mathrm{PH}$ & NS & ${ }^{* *}(+)$ & NS & $* \star(-)$ & ${ }^{*}\left(\_\right)$ & NS & NS & 1,000 & \\
\hline CE & NS & NS & NS & NS & NS & ${ }^{* *}(+)$ & NS & NS & 1,000 \\
\hline
\end{tabular}

* Correlación significativa $(P<0,05)$

** Correlación altamente significativa $(P<0,01)$

NS: Correlación no Significativa $(P>0,05)$

En la Tabla 4, se observa que el pH de los suelos, correlacionó de manera positiva y y no significativa con la producción de taninos condensados y altamente significativa con la concentración de $\mathrm{TH}$, pero presentó correlación negativa altamente significativa con los TPP.. Lo anterior coincide con lo planteado por Hagerman et al. (1998) al exponer que los taninos no precipitan proteína a pH muy altos. Al respecto Ramos et al. (1998), citando a Koupai - Abyazani (1993), expresan que los complejos tanino-proteína se forman con mayor facilidad a un $\mathrm{pH}$ próximo a 6,0 , correspondiente a los valores medios en el rumen.

Se detectó que el nitrógeno amoniacal correlacionó de forma negativa y muy significativa con la concentración de taninos hidrolizables y con los taninos que precipitan proteína. El Nitrógeno nítrico no afectó de manera significativa el con- tenido de taninos condensados, TH, FT, TPP, en Lotus curniculatus. Finalmente, se destacó la correlación negativa y de alta significancia estadística entre la capacidad de intercambio catiónica efectiva de los suelos y la producción de taninos condensados y fenoles totales en la planta estudiada. No se encontraron correlaciones significativas entre la conductividad eléctrica y los metabolitos secundarios estudiados, y ello no coincide con lo reportado por Arambula, Ibarra, González, Galindo \& Hernández (2010), quien había identificado una correlación alta y positiva entre el contenido fenólico y la conductividad eléctrica en suelos de textura arcillo-arenosa. En el mismo sentido, Santacoloma \& Granados (2012) hallaron una alta correlación entre el valor de la conductividad eléctrica del suelo y el contenido de metabolitos secundarios en las especies Gliricidis sepium y Tithonia diversifolia. 


\section{Saponinas}

Los resultados de la presencia de saponinas en las muestras de forraje de los tres tratamientos realizada a través de análisis cualitativo se presentan en la Tabla 5:

Tabla 5.Resultados obtenidos en la determinación de saponinas

\begin{tabular}{lcc}
\hline Tratamiento & $\begin{array}{c}\text { Nivel de } \\
\text { espuma }\end{array}$ & $\begin{array}{c}\text { Nivel de } \\
\text { espuma }\end{array}$ \\
\hline Tratamiento 1 & $3 \mathrm{~mm}$ & $4 \mathrm{~mm}$ \\
\hline Tratamiento 2 & $4 \mathrm{~mm}$ & $4 \mathrm{~mm}$ \\
\hline Tratamiento 3 & $5 \mathrm{~mm}$ & $4 \mathrm{~mm}$ \\
\hline
\end{tabular}

Según el análisis cualitativo no se identificó la presencia de saponinas en las muestras, Estos compuestos, al igual que los taninos en concentraciones óptimas, podrían tener incidencia en la mejora de la eficiencia en la utilización del alimento en rumiantes, aumentando el flujo de proteína microbiana hacia el duodeno (Makkar et al., 1997). Al respecto Abreu et al. (2004) encontraron en frutos con contenidos de saponinas una reducción de la población de protozoarios, lo cual puede redundar en una utilización eficiente de la fibra.

\section{Alcaloides}

En el ensayo no se reportó presencia de alcaloides, metabolitos frecuentes dentro de las leguminosas, que le confieren un sabor amargo y algunas características de toxicidad. En la Tabla 6 se presentan los resultados obtenidos:

Tabla 6. Resultados obtenidos en la determinación de alcaloides

\begin{tabular}{lcc}
\hline \multicolumn{1}{c}{ Tratamiento } & Color & Presencia \\
\hline Tratamiento 1 & Amarillo oscuro-café & No \\
Tratamiento 2 & Amarillo oscuro-café & No \\
Tratamiento 3 & Amarillo oscuro-café & No \\
\hline
\end{tabular}

\section{Conclusiones}

Los resultados permiten concluir, que en suelos de buenas características de fertilidad se presenta una disminución en la concentración de metabolitos secundarios, en la especie Lotus corniculatus. Desde el punto de vista nutricional, esto tiene alta relevancia, ya que basado en las cuantificaciones realizadas, la especie cultivada en un suelo de óptima fertilidad presenta buena calidad nutricional para ser empleada en la alimentación de rumiantes, debido a las bajas concentraciones de compuestos con potencialidades tóxicas. Lo anterior, se sustenta en que los taninos en concentraciones que van de bajas a moderadas previenen timpanismos e incrementan el flujo de nitrógeno no amoniacal y de aminoácidos esenciales del rumen.

Los contenidos de Taninos Condensados fueron intermedios, aunque ligeramente altos para el forraje presente en suelos poco fértiles, estos últimos son cercanos a valores que pueden afectar la fermentación en el rumen, no obstante, constituyen valores que coinciden, de manera general, con los valores de la fracción comestible de leguminosas típicas utilizadas para la alimentación animal. 
Las relaciones entre el suelo y el metabolismo secundario de las plantas constituyen un factor importante para disminuir el efecto adverso que tienen algunos forrajes sobre la digestión y absorción de nutrientes en rumiantes

Las evaluaciones de recursos forrajeros, mediante la determinación de la concentración de compuestos secundarios, como variables asociadas al valor nutritivo, resultan de vital importancia en la caracterización integral de recursos forrajeros para animales rumiantes

\section{Literatura citada}

1. Abreu, A., Carulla, J. E., Lascano, C. E., Diaz, T. E., Kreuzer, M., \& Hess, H. D. (2004). Effects of Sapindus saponaria fruits on ruminal fermentation and duodenal nitrogen flow of sheep fed a tropical grass diet with and without legume. Journal of animal science, 82(5), 1392-1400.

2. Andrabi, S.M., Ritchie, M.M., Stimson, C., Horadagoda, A, Hyde, M., \& McNeill,D.M. (2005).In vivo assessment of the ability of condensed tannins to interfere with the digestibility of plant protein in sheep. Animal feed Science and Technology 122:13-27

3. Arambula, J., Ibarra, B.I., Gonzalez, B., Galindo, O.D., \& Hernández, H. (2010). Variación estacional de compuestos fenólicos foliares en Quersus sideroxila en diferentes tipos de suelos. Madera y bosques 16 (3).4959. Instituto de ecología. AC, Xalapa (México)

4. Ashok, K. .J., Vincent, R. M. \& Nessler (2001). Molecular Characterization of a hydroxyl Methylglutaryl Coareductase gene from Mulberry (Morus Alba L). Plant mol Biol 42:559

5. Barahona Rosales, R., Theodoru, M., Morris, P., Owen, E., \& Lascano, C. E. (1997). Avances en la investigación de factores antinutricionales en leguminosas forrajeras tropicales.

6. Barry T N \& Mc Nabb W C (1999). The Effect of Condensed Tannins in Temperate forages on Animal Nutrition and Produc-tivity. In Tannins in Livestock and Human Nutrition, pp 30-35 [J D Brooker, editor]. Canberra Australian Center for In-ternational Agricultural Research. http://www.aciar.gov.au/.

7. Berard, N. C., Wang, Y., Wittenberg, K. M., Krause, D. O., Coulman, B. E., McAllister, T. A., \& Ominski, K. H. (2011). Condensed tannin concentrations found in vegetative and mature forage legumes grown in western Canada. Canadian Journal of Plant Science, 91(4), 669-675.
8. Beecher, G., Gu, L., Kelm, M. A., Hammerstone, J. F.,., Holden, J., Haytowitz, D., \& Prior, R. L. (2004). Concentrations of proanthocyanidins in common foods and estimations of normal consumption. The Journal of nutrition, 134(3), 613-617.

9. Bueno, I., Vitti, D., louvandini, H., \& Abdala, A. (2008). A ney approach for in vitro bioassay to measure tannin biological effects based on a gas production technique. Animal feed Science and Technology 141: 153-170

10. Caviedes, J., .Pabón, L. \& Carulla, J. (2011). Relación entre las características de la pastura y el contenido de ácido linoleico conjugado (ALC) en la leche. Revista Colombiana de Ciencias Pecuarias. 24:63-73. Medellín

11. Galindo, W.F, Rosales, M., Murgueitio, E. \& Larrahondo, J. (1989). Sustancias antinutricionales en las hojas de guamo, nacedero y matarratón. Livestock Research for Rural Development, 1 (1) 36-47.

12. García, D. E., Medina, M. G., Ojeda, F., Humbría, J., Domínguez, C., Baldizán, A., \& Toral, O. (2007). Variabilidad fitoquímica y repercusión antinutricional potencial en especies del género Albizia. Pastos y Forrajes, 30(1), 1-1.

13. Hagerman, A. E., Riedl, K. M., Jones, G. A., Sovik, K. N., Ritchard, N. T., Hartzfeld, P. W., \& Riechel, T. L. (1998). High molecular weight plant polyphenolics (tannins) as biological antioxidants. Journal of Agricultural and Food Chemistry, 46(5), 1887-1892.

14. Hartzfeld P. W.; Forkner R.; Hunter M. D. \& Hagerman A E. (2002) Determination of hydrolyzable tannins (gallotannins and ellagitannins) after reaction with potassium iodate. J. Agric. Food Chem. 50(7): 1785-1790.

15. Jungk A. (2001) Root hairs and the acquisition of plant nutrients from soil. J. Plant Nutr. Soil Sci. 162:9-24

16. Khiaosa-Ard R, Bryner DF, Scheeder MRL, Wettstein HR, Leiber F, Kreuzer M, and Soliva C.R .(2009). Evidence for the inhibition of the terminal step of ruminal linolenic acid biohydrogenation by condensed tannins. J Dairy Sci ; 92:177-188.

17. Koupai-Abyazani, M. R., Muir, A. D., Bohm, B. A., Towers, G. H., \& Gruber, M.Y. (1993). The proanthocyanidin polymers in some species of Onobrychis. Phytochemistry, 34(1), 113-117.

18. Kumar, R. (1997). Ant nutritional factors. The potential risks of toxicity and the methods to alleviate them. In Legumes trees and other fodder trees as protein source for livestock. (Eds. Speedy).

19. Leinmúller, E., H, Steingass, H \& Menke, K.H:. (1995). Tannins in ruminant feedstuffs. Animal and Research and Develovment 33: 9-62

20. Makkar, H. P., Blümmel, M. \& Becker, k. (1997). Formation of complexes between polyvinyl pyrrolidones or polyethylene glycols and tannins, and their implication in gas production and true digestibility in vitro techniques. British Journal of Nutrition. 73: 897-913.

21. Makkar, H. P. \& Goodchild, V. A. (1996). Quantification of tannins a laboratory manual. pasture, forage and livestock program International Center for Agricultural research in the dry areas. Second edition. Alepo, Syria. 
22. Márquez, D. \& Suárez, A. (2008). El uso de taninos condensados como alternativa nutricional y sanitaria en rumiantes. Revista de Medicina Veterinaria Nro. 16 / julio - diciembre 2008. 87-109

23. Min, B.R., Attwood, G.T., McNabb, W.C., Molan, A.L. \& Barry, T.N. (2005). The effect of condensed tannins from Lotus corniculatus on the proteolytic activities and growth of rumen bacteria. Animal Feed Science and Technology 121: 45-58.

24. Mueller-Harvey, I. (2001). Analysis of hydrolysable tannins. Animal Feed Science and Technology 91(1), 3-20.

25. Norton, B.W. (1999). The significance of Tannins in Tropical Animal Production. CIAR Procedings No. 92, Canberra, Australia, 14-23.

26. Otero, M. \& Hidalgo, L. (2004). Condensed tannins in temperate forages species: effects on the productivity of ruminants infected with internal parasites (a review). Livestock Research for Rural Development 16(2): 18-36

27. Posada, S., Montoya, G. \& Ceballos, A. (2005). Efecto de los taninos sobre la digestión, el metabolismo y la producción en rumiantes" Bioquímica, Nutrición y Alimentación De La Vaca . En: Colombia ISBN: 9583372773 ed: Biogénesis, v. p. $181-206$.

28. Provenza, F.D. Burrit, E.A., Perevolotsky, A. \& Silanokove, N. (2000). Self regulation of intake of polyethylene by sheep fed diets varying in tannin concentrations. Journal Animal Science 78: 1206-1212

29. Ramos, G., Frutos, P., Giráldez, F. J., \& Mantecón, Á. R. (1998). Los compuestos secundarios de las plantas en la nutrición de los herbívoros. Archivos de zootecnia, 47, 597-620.

30. Robbins, M. P., Bavage, A. D., Allison, G., Davies, T., Hauck, B. \& Morris, P. (2005). A comparison of two strategies to modify the hydroxylation of condensed tannin polymers in Lotus corniculatus y L. Phytochemistry, 66(9), 991-999.

31. Rojas, D.K., López, J, Tejada, I., Vásquez, V., Shimada, A., Sánchez, D. \& Ibarra, F. (2006). Impacto f condensed tannins from trpoical forages on Haemonchus contortus burdens in Mongolia gerbils (Meriones unguiculatus) and pelibuey lambs. Animal Feed Science and Technology 128: 218-228.

32. Rubanza, C. D., Shem. M. N., Otsyina, R., Bakengesa, S. S., Ichinohe, T. \& Fujihara, T. (2005). Polyphenolics and tannins effect on in vitro digestibility of selected Acacia species leaves. Animal Feed Science and Technology 119: 129-142.
33. Santacoloma, L. E. \& Granados, J. (2012). Interrelación entre el contenido de metabolites secundarios de lase species Gliricidia sepium y Tithonia diversifolia y algunas propiedades físicoquímicas del suelo. Revista de Investigación Agraria y Ambiental, 3 (1); 53-62.

34. Stürm, C.D., Tiemann, T.T., Lascano, C.E., Creuzer, M. \& Hess, H.D. (2007). Nutrient composition and in vitro ruminal fermentation of tropical legume mixtures with contrasting tannin contents. Animal Feed Science and Technology 138: 29-46.

35. Terril, T. H., Rowan, A.M., Douglas. G.B. \& Barry,T.N (1997) .Determination of extractable and bound condensed tannin concentration in forage plants, protein concentrate meals and cereal grains. J.Sci. Food agric.58:321-329.

36. Thévenot, M., Dignac, M. F. \& Rumpel, C. (2010). Fate of lignins in soils: a review. Soil Biol. Biochem. 42:12001211.

37. Troiani, A., Rosi, M., Angelini, G., \& Ursini, O. (2009). Methane Activation by Metal-Free Radical Cations: Experimental Insight into the Reaction Intermediate. Chemistry-A European Journal, 15(17), 4248-4252

38. Vilela, A. E., González-Paleo, L. \& Ravetta, D. A. (2011). Metabolismo secundario de plantas leñosas de zonas áridas: mecanismos de producción, funciones y posibilidades de aprovechamiento. Ecología austral, 21(3), 317-327.

39. Waghorn, C. G., Reed, J. D. \& Ndlovu, L. R. (1997) Condensed Tannins and Herbivore Nutrition. Sección 8- Tannins Plant Bre-eding and Animal Effects. Proceedings of theXVIII . International Grassland Congress. Canada, 1997.

40. Waghorn \& McNabb, (2003). Effect of condensed tannins in Lotus corniculatus on the nutritive value of pasture for sheep. Journal of Agricultural Science, Cambridge, 128, 365-372.

41. Wang, Y., Douglas, G. B., Waghorn, C. G., Barry, T. N., Foote, A. G. \& Purchas, R. W. (1996) Effect of condensed tannins upon the performance of lambs grazing Lotus corniculatus and Lucerne (Medicago sativa). Journal of Agricultural Science, Cam-bridge, 126:87-98.

42. Waterman, P. G. \& Mole, S. (1995) "Analysis of Phenolic Plant metabolites," Blackwell Scientific Publications, Ed., Cambridge. MA, USA Págs 67-13 